Ophthalmologe 2018 $\cdot 115: 529$

https://doi.org/10.1007/s00347-018-0711-3

Online publiziert: 30. April 2018

(c) Springer Medizin Verlag GmbH, ein Teil von Springer Nature 2018

CrossMark

Ulrike Pichler

Med. Campus III, Klinik für Augenheilkunde, Orthoptik, Kepler Universitätsklinikum, Linz, Österreich

\title{
Kontroversen in der Prismenvorbehandlung vor Schieloperation
}

convergent squint“. Dieser Artikel wurde dankenswerterweise von Fr. Prof. Stangler-Zuschrott zur Verfügung gestellt, da er für uns nicht zugänglich war.

Die sehr interessante Arbeit von Zehetmayer et al. [2] vergleicht die Operationsergebnisse nach mehrmonatiger vs. keine Prismenadaptation. Somit ist unsere Aussage weiterhin richtig, dass keine Studie kurz- vs. langzeitige Prismenadaptation vergleicht. Leider handelt es sich auch um eine retrospektive, nicht randomisierte Studie. Da Kontroll- und Therapiegruppe sicherlich unterschiedlich sind, müssen leider viele der getroffenen Aussagen mit Vorsicht interpretiert werden.

Ad Punkt 5) Das Thema der Stigmatisierung wurde bereits im Artikel behandelt. Zudem sollte auch bedacht werden, dass trotz preisgünstiger Brillen oder Folien trotzdem Kosten anfallen, die nicht von allen so leicht getragen werden können. Ferner sind die Kosten für die häufigeren Untersuchungen (Wegekosten, Arbeitszeitausfall) als nicht unerheblich anzusehen. Die Datenlage zeigt, dass früher im Schnitt 14 Sehschulbesuche wahrgenommen wurden, ehe eine Schieloperation stattfand.

Ad Punkt 6) Uns ist keine prospektive randomisierte Studie bekannt, die die Aussage „Prismentherapie reduziert wesentlich die Zahl der Schieloperationen“" belegt. Auch ohne Prismentherapie kann sich bei vielen Kindern durch mehrjähriges Abwarten die Notwendigkeit einer Schieloperation erübrigen (z.B. ELISSStudie).

\section{Korrespondenzadresse}

\section{U. Pichler, M.Sc}

Med. Campus III, Klinik für Augenheilkunde, Orthoptik, Kepler Universitätsklinikum Krankenhausstr. 9, 4020 Linz, Österreich ulrike.pichler@kepleruniklinikum.at

Interessenkonflikt. U. Pichler gibt an, dass kein Interessenkonflikt besteht.

\section{Literatur}

1. Neikter B (1994) Horizontal and vertical deviation after prism neutralization and diagnostic occlusion in intermittent exotropia. Strabismus 2(1):13-22

2. Zehetmayer $M$, Stangler-Zuschrott $E$, Schemper M (1994) Prolonged preoperative prismatic treatment in alternating convergent squint. Acta Ophthalmol 72:103-109

3. Stangler-Zuschrott E (2012) Prismen zur Behandlung des Strabismus bei Kindern. Spektrum Augenheilkd 26:230-235 